Article

\title{
Generalized Bertrand Curves in Minkowski 3-Space
}

\author{
Chunxiao Zhang and Donghe Pei *(D) \\ School of Mathematics and Statistics, Northeast Normal University, Changchun 130024, China; \\ zhangcx243@nenu.edu.cn \\ * Correspondence: peidh340@nenu.edu.cn
}

Received: 19 October 2020; Accepted: 8 December 2020; Published: 10 December 2020

\begin{abstract}
We define a generalized lightlike Bertrand curve pair and a generalized non-lightlike Bertrand curve pair, discuss their properties and prove the necessary and sufficient condition of a curve which is a generalized lightlike or a generalized non-lightlike Bertrand curve. Moreover, we study the relationship between slant helices and generalized Bertrand curves.
\end{abstract}

Keywords: lightlike Bertrand curves; non-lightlike Bertrand curves; generalized Bertrand curves pair

\section{Introduction}

The Bertrand curve is widely studied by many mathematicians in classical differential geometry. It was first proposed by Bertrand who supported that a curve is a Bertrand curve in $\mathbb{R}^{3}$ if and only if the curvature $\kappa$ and the torsion $\tau$ satisfy $\eta \kappa+\mu \tau=1$, where $\eta \neq 0, \mu$ are constants. Bertrand curves have been studied by many mathematicians in various spaces. For instance, in [1], J. Huang and D. Pei studied the singular Bertrand curves in the 3-dimensional space forms. In [2], Takeuchi and Izumiya studied the general properties of helice and Bertrand curve, and presented the method of constructing a Bertrand curve from the spherical curve. In [3], Ekmekçi and İlarslan defined a Bertrand curve in n-dimensional Lorentz space and obtained some properties on this space. In [4], some properties of the non-lightlike curve in 3-dimensional Lorentz space were given. Then, in [5,6], Balgetir and Honda studied the lightlike Bertrand curves and gave their geometric properties. For non-flat space, such as the 3-dimensional sphere $S^{3}$, Lucas and Yagües defined a new Bertrand curve $[7,8]$. They considered the correspondence of the principal normal geodesics by using the tools of connection, and gave the relationship between $(1,3)$-type Bertrand curve in $\mathbb{R}^{4}$ and the Bertrand curve on 3-dimensional sphere $S^{3}$. Moreover, in other spaces, such as in Riemann-Otsuki spaces [9], and on 3-dimensional Riemannian manifolds $[10,11]$, some scholars also studied the theory of Bertrand curves.

The Bertrand curve can be regarded as the generalization of the helix. The helix, as a special kind of curve, has drawn the attention of scientists as well as mathematicians because of its various applications, for example, explanation of DNA, carbon nano-tube, nano-springs, a-helices, the geometrical shaping of linear chained polymers stabilized as helixes and the eigenproblem translated for alignment of molecules (see [12-15]). Moreover, the Bertrand curves represent particular examples of offset curves which are used in computer-aided design (CAD) and computer-aided manufacture (CAM) (see [16,17]). The effective use of road edge information in high-resolution remote sensing image plays an important role in improving the accuracy of road extraction, and the road edge in high-resolution remote sensing image has obvious characteristics of the Bertrand curves (see [18]). Therefore, there are potential implications for their research. In the early 20th century, the German mathematician Minkowski proposed Minkowski space firstly. Since the theory of relativity was put forward by Einstein, Minkowski space, as a space-time model, has been widely concerned by mathematical and physical circles. As a pseudo Euclidean space, the measurement of Minkowski space is different from Euclidean metric, so the results of many problems in these two spaces are 
also different. The curves in Minkowski space can be divided into two categories: non-lightlike curves and lightlike curves. The second author, J. Sun and some others introduced the properties of non-lightlike curves in Minkowski 3-space. Bonner introduced the pseudo arc length parameter in [19] and proved the existence and uniqueness of the lightlike curve. In this paper, we mainly study the the generalization of Bertrand curves in Minkowski 3-space.

We suppose here that all manifolds and maps are smooth.

\section{Preliminaries}

Let $\mathbb{R}^{3}=\left\{\left(p_{1}, p_{2}, p_{3}\right) \mid p_{1}, p_{2}, p_{3} \in \mathbb{R}\right\}$ be a 3-dimensional vector space. For $\boldsymbol{p}=\left(p_{1}, p_{2}, p_{3}\right)$, $\boldsymbol{q}=\left(q_{1}, q_{2}, q_{3}\right) \in \mathbb{R}^{3}$, the pseudo inner product between $\boldsymbol{p}$ and $\boldsymbol{q}$ is

$$
\langle\boldsymbol{p}, \boldsymbol{q}\rangle=-p_{1} q_{1}+p_{2} q_{2}+p_{3} q_{3}
$$

we call $\left(\mathbb{R}^{3},\langle\rangle,\right)$ is the Minkowski 3-space $\mathbb{R}_{1}^{3}$.

For $\boldsymbol{p}=\left(p_{1}, p_{2}, p_{3}\right), \boldsymbol{q}=\left(q_{1}, q_{2}, q_{3}\right) \in \mathbb{R}_{1}^{3}$, the pseudo vector product between $\boldsymbol{p}$ and $\boldsymbol{q}$ is

$$
\boldsymbol{p} \wedge \boldsymbol{q}=\left|\begin{array}{ccc}
-\mathbf{e}_{1} & \mathbf{e}_{2} & \mathbf{e}_{3} \\
p_{1} & p_{2} & p_{3} \\
q_{1} & q_{2} & q_{3}
\end{array}\right|
$$

where $\left(\mathbf{e}_{1}, \mathbf{e}_{2}, \mathbf{e}_{3}\right)$ is a canonical basis of $\mathbb{R}_{1}^{3}$.

We call a non-zero vector $\boldsymbol{p} \in \mathbb{R}_{1}^{3}$ spacelike, lightlike or timelike if $\langle\boldsymbol{p}, \boldsymbol{p}\rangle>0,\langle\boldsymbol{p}, \boldsymbol{p}\rangle=0$ or $\langle\boldsymbol{p}, \boldsymbol{p}\rangle<0$, respectively.

We call a curve $\mathbf{c}(t)$ in $\mathbb{R}_{1}^{3}$ spacelike, lightlike or timelike if $c^{\prime}(t)$ is spacelike, lightlike or timelike, respectively.

The sign function $\delta(\boldsymbol{p})$ of $\boldsymbol{p}$ is 1,0 or -1 if $\boldsymbol{p}$ is spacelike, lightlike or timelike, respectively.

Let $\mathbf{c}(s)$ be a non-lightlike(spacelike or timelike) curve in $\mathbb{R}_{1}^{3}$, we suppose that it is parametrized by arc-length $s$, if

$$
\langle\ddot{\mathbf{c}}(s), \ddot{\mathbf{c}}(s)\rangle \neq 0 .
$$

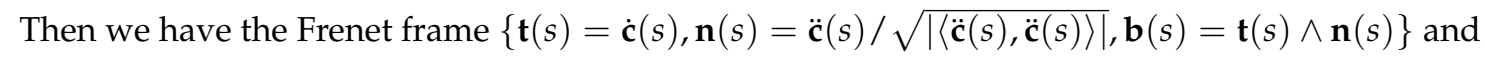
the Frenet equations (see [20]):

$$
\left\{\begin{array}{l}
\dot{\mathfrak{t}}(s)=\kappa(s) \mathbf{n}(s) \\
\dot{\mathbf{n}}(s)=-\delta(\mathbf{t}) \delta(\mathbf{n}) \kappa(s) \mathfrak{t}(s)+\delta(\mathbf{t}) \tau(s) \mathbf{b}(s) \\
\dot{\mathbf{b}}(s)=\tau(s) \mathbf{n}(s)
\end{array}\right.
$$

where

$$
\kappa(s)=\langle\dot{\mathbf{t}}(s), \mathbf{n}(s)\rangle, \tau(s)=\langle\mathbf{n}(s), \dot{\mathbf{b}}(s)\rangle .
$$

We call $\kappa(s), \tau(s), \mathbf{t}(s), \mathbf{n}(s)$ and $\mathbf{b}(s)$ the curvature, torsion, tangent, principal normal and binormal of $\mathbf{c}(s)$, respectively.

The Darboux vector field $\mathbf{d}(s)$ along any non-lightlike curve $\mathbf{c}(s)$ is $\mathbf{d}(s)=-\delta(\mathbf{t}) \delta(\mathbf{n}) \tau(s) \mathbf{t}(s)+$ $\delta(\mathbf{t}) \delta(\mathbf{n}) \kappa(s) \mathbf{b}(s)$.

Let $\mathbf{c}(t)$ be a lightlike curve in $\mathbb{R}_{1}^{3}$. We can get that $\frac{d^{2} c}{d t^{2}}$ is spacelike, and give a reparametrization $s=s(t)$ of $\mathbf{c}$ such that

$$
\langle\ddot{\mathbf{c}}(s), \ddot{\mathbf{c}}(s)\rangle=1,
$$

where

$$
\ddot{\mathbf{c}}=\frac{d^{2} \mathbf{c}}{d s^{2}} \text {. }
$$


$s$ is called the pseudo arc length parameter of $\mathbf{c}$. Let

$$
\mathfrak{t}(s)=\dot{\mathbf{c}}(s), \mathbf{n}(s)=\dot{\mathbf{t}}(s),
$$

then we can get the unique vector field $\mathbf{b}(s)$, such that $\{\mathbf{t}(s), \mathbf{n}(s), \mathbf{b}(s)\}$ forms a lightlike frame along $\mathbf{c}(s)$, and we have the lightlike Frenet equations (see [21]):

$$
\left\{\begin{array}{l}
\dot{\mathfrak{t}}(s)=\mathbf{n}(s) \\
\dot{\mathbf{n}}(s)=-\tau(s) \mathbf{t}(s)-\mathbf{b}(s) \\
\dot{\mathbf{b}}(s)=\tau(s) \mathbf{n}(s)
\end{array}\right.
$$

where

$$
\tau(s)=\langle\dot{\mathbf{b}}(s), \mathbf{n}(s)\rangle .
$$

The lightlike frame $\{\mathbf{t}(s), \mathbf{n}(s), \mathbf{b}(s)\}, \mathbf{c}(s)$ and $\tau(s)$ is called the Cartan frame, the lightlike Cartan curve and the lightlike curvature of $\mathbf{c}(s)$, respectively.

The Darboux vector field $\mathbf{d}(s)$ along lightlike Cartan curve $\mathbf{c}(s)$ is $\mathbf{d}(s)=\tau(s) \mathbf{t}(s)+\mathbf{b}(s)$.

For more details, see [20,21].

\section{Generalized Bertrand Curves in Minkowski 3-Space}

In [8], Lucas and Ortega-Yagües defined the angle between two vectors in $\mathbb{R}_{1}^{3}$. Then we have the following.

\subsection{Generalized Non-Lightlike Bertrand Curves in Minkowski 3-Space}

Definition 1. Let $\boldsymbol{c}(s)$ and $\overline{\boldsymbol{c}}(\overline{\mathrm{s}})$ be two non-lightlike curves in $\mathbb{R}_{1}^{3}$. If the principal normal $\boldsymbol{n}(s)$ of $\boldsymbol{c}(s)$ lies in the normal plane of $\overline{\boldsymbol{c}}(\overline{\boldsymbol{s}})$ and the angle between $\boldsymbol{n}(s)$ and $\overline{\boldsymbol{n}}(\overline{\boldsymbol{s}})$ is $\theta$ at the corresponding points, then we call $\boldsymbol{c}(s)$ a generalized Bertrand curve, $\overline{\boldsymbol{c}}(\bar{s})$ a generalized Bertrand mate of $\boldsymbol{c}(s)$ and $(\boldsymbol{c}(s), \overline{\boldsymbol{c}}(\overline{\mathrm{s}})$ a pair of generalized Bertrand curves.

Without considering the lightlike case, Frenet frame in $\mathbb{R}_{1}^{3}$ only has three cases: (1) $\mathfrak{t}(s)$ and $\mathbf{n}(s)$ are spacelike, $\mathbf{b}(s)$ is timelike. (2) $\mathbf{t}(s)$ and $\mathbf{b}(s)$ are spacelike, $\mathbf{n}(s)$ is timetlike. (3) $\mathbf{b}(s)$ and $\mathbf{n}(s)$ are spacelike, $\mathbf{t}(s)$ is timetlike.

Theorem 1. Let $\left(\boldsymbol{c}(s), \overline{\boldsymbol{c}}(\bar{s})\right.$ be a pair of Bertrand curves in $\mathbb{R}_{1}^{3}$, then we have:

(1) If $\boldsymbol{c}(s)$ is a spacelike curve with $\boldsymbol{n}(s)$ being spacelike and $\boldsymbol{b}(s)$ being timelike, then when $|1-\eta \kappa(s)|<$ $|\eta \tau(s)|$, Bertrand mate $\overline{\boldsymbol{c}}(\bar{s})$ is a timelike curve, when $|1-\eta \kappa(s)|>|\eta \tau(s)|$, Bertrand mate $\overline{\boldsymbol{c}}(\bar{s})$ is a spacelike curve.

(2) If $\boldsymbol{c}(s)$ is a spacelike curve with $\boldsymbol{n}(s)$ being timelike and $\boldsymbol{b}(s)$ being spacelike, then Bertrand mate $\overline{\boldsymbol{c}}(\overline{\boldsymbol{s}})$ is a spacelike curve.

(3) If $\boldsymbol{c}(s)$ is a timelike curve with $\boldsymbol{n}(s)$ and $\boldsymbol{b}(s)$ being spacelike, then when $|1+\eta \kappa(s)|>|\eta \tau(s)|$, Bertrand mate $\overline{\boldsymbol{c}}(\bar{s})$ is a timelike curve, when $|1+\eta \kappa(s)|<|\eta \tau(s)|$, Bertrand mate $\overline{\boldsymbol{c}}(\bar{s})$ is a spacelike curve, where $\eta$ is the distance between $c(s)$ and $\overline{\boldsymbol{c}}(\bar{s})$ at the corresponding points and $\eta$ is a non-zero constant.

Proof. (1) As c(s) is a Bertrand curve, we can get

$$
\overline{\mathbf{c}}(\bar{s})=\mathbf{c}(s)+\eta(s) \mathbf{n}(s),
$$

where $\bar{s}=\bar{s}(s)$, differentiating it with respect to $s$, we will have

$$
\left.\overline{\mathbf{t}}(\bar{s}) \frac{d \bar{s}}{d s}=[(1-\eta \kappa) \mathbf{t}+\dot{\eta} \mathbf{n}+\eta \tau \mathbf{b})\right](s) .
$$


Taking the pseudo inner product with $\overline{\mathbf{n}}(\bar{s})$ on both sides it, we have

$$
\dot{\eta}(s)=0,
$$

so

$$
\langle\overline{\mathbf{t}}(\bar{s}), \overline{\mathbf{t}}(\bar{s})\rangle=\left[(1-\eta \kappa(s))^{2}-\eta^{2} \tau^{2}(s)\right]\left(\frac{d s}{d \bar{s}}\right)^{2} .
$$

Thus, when $|1-\lambda \kappa(s)|<|\eta \tau(s)|$, Bertrand mate $\overline{\mathbf{c}}(\bar{s})$ is a timelike curve, when $|1-\lambda \kappa(s)|>$ $|\eta \tau(s)|$, Bertrand mate $\overline{\mathbf{c}}(\bar{s})$ is a spacelike curve. We can prove (2), (3) in a similar way. This completes the proof.

Theorem 2. Let $\boldsymbol{c}(s)$ be a spacelike curve with $\boldsymbol{n}(s)$ being spacelike and $\boldsymbol{b}(s)$ being timelike, $\overline{\boldsymbol{c}}(\overline{\boldsymbol{s}})$ be a timelike curve with $\overline{\boldsymbol{n}}(\bar{s})$ and $\overline{\boldsymbol{b}}(\overline{\mathrm{s}})$ are spacelike, then $(\boldsymbol{c}(s), \overline{\boldsymbol{c}}(\overline{\mathrm{s}}))$ is a pair of generalized Bertrand curves if and only if the curvature $\kappa(s)$ and torsion $\tau(s)$ of $c(s)$ satisfy

$$
\left[\kappa-\eta\left(\kappa^{2}-\tau^{2}\right)\right](s)=\cos \theta \frac{\left\{\left[\kappa-\eta\left(\kappa^{2}-\tau^{2}\right)\right]^{2}\left[\eta^{2} \tau^{2}-(1-\eta \kappa)^{2}\right]+\left[\eta \dot{\tau}-\eta^{2}(\kappa \dot{\tau}-\dot{\kappa} \tau)\right]^{2}\right\}^{\frac{1}{2}}}{\left[\eta^{2} \tau^{2}-(1-\eta \kappa)^{2}\right]^{\frac{1}{2}}}(s),
$$

where $\theta$ is the angle between $\boldsymbol{n}(s)$ and $\overline{\boldsymbol{n}}(\bar{s}), \eta$ is a non-zero constant, $\dot{\kappa}=\frac{d \kappa}{d s}$ and $\dot{\tau}=\frac{d \tau}{d s}$.

Proof. As $(\mathbf{c}(s), \overline{\mathbf{c}}(\bar{s}))$ is a pair of generalized Bertrand curves, we obtain

$$
\overline{\mathbf{c}}(\bar{s})=\mathbf{c}(s)+\eta(s) \mathbf{n}(s),
$$

where $\bar{s}=\bar{s}(s), \eta(s)$ is the distance at the corresponding points between $\mathbf{c}(s)$ and $\overline{\mathbf{c}}(\bar{s})$.

When $\overline{\mathbf{c}}(\bar{s})$ is timelike curve, its normal plane is spacelike, the angle between $\mathbf{n}(s)$ and $\overline{\mathbf{n}}(\bar{s})$ is $\theta$, so

$$
\mathbf{n}(s)=\cos \theta \overline{\mathbf{n}}(\bar{s})+\sin \theta \overline{\mathbf{b}}(\bar{s}) .
$$

Differentiating Equations (1) and (2) with respect to $s$, we have

$$
\begin{gathered}
\overline{\mathbf{t}} \frac{d \bar{s}}{d s}(\bar{s})=(\mathbf{t}+\dot{\eta} \mathbf{n}+\eta \dot{\eta} \dot{\mathbf{n}})(s), \\
\dot{\mathbf{n}}(s)=\left[\cos \theta \dot{\overline{\mathbf{n}}} \frac{d \bar{s}}{d s}+\sin \theta \dot{\overline{\mathbf{b}}} \frac{d \bar{s}}{d s}\right](\bar{s}) .
\end{gathered}
$$

We have known

$$
\dot{\eta}(s)=0,
$$

then we can get

$$
\overline{\mathbf{t}} \frac{d \bar{s}}{d s}(\bar{s})=[(1-\eta \kappa) \mathbf{t}+\eta \tau \mathbf{b}](s) .
$$

Using the Frenet equations, we get

$$
\begin{gathered}
(-\kappa \mathbf{t}+\tau \mathbf{b})(s)=\left[\bar{\kappa} \cos \theta \frac{d \bar{s}}{d s} \overline{\mathbf{t}}+\sin \theta \bar{\tau} \frac{d \bar{s}}{d s} \overline{\mathbf{n}}-\bar{\tau} \cos \theta \frac{d \bar{s}}{d s} \overline{\mathbf{b}}\right](\bar{s}) . \\
{\left[\kappa-\eta\left(\kappa^{2}-\tau^{2}\right)\right](s)=\bar{\kappa} \cos \theta\left(\frac{d \bar{s}}{d s}\right)^{2}(\bar{s}) .}
\end{gathered}
$$

The curvature $\bar{\kappa}(\bar{s})$ of curve $\overline{\mathbf{c}}(\bar{s})$ is

$$
\bar{\kappa}(\bar{s})=\frac{|\dot{\overline{\mathbf{c}}} \wedge \ddot{\overline{\mathbf{c}}}|}{|\dot{\mathbf{c}}|^{3}}(\bar{s}),
$$


where $\dot{\overline{\mathbf{c}}}=\frac{d \overline{\bar{c}}}{d s}$, so

$$
\begin{gathered}
\dot{\overline{\mathbf{c}}}(\bar{s})=\overline{\mathbf{t}} \cdot \frac{d \bar{s}}{d s}(\bar{s}), \\
|\dot{\overline{\mathbf{c}}}|=\left|\frac{d \bar{s}}{d s}\right| .
\end{gathered}
$$

We put Equations (7) and (9) in Equation (6), then

$$
\left[\kappa-\eta\left(\kappa^{2}-\tau^{2}\right)\right](\bar{s})=\cos \theta \frac{|\dot{\overline{\mathbf{c}}} \wedge \ddot{\overline{\mathbf{c}}}|}{|\overline{\dot{\mathbf{c}}}|}(\bar{s})
$$

and by using Equations (4) and (8), we can see

$$
\dot{\overline{\mathbf{c}}}(\bar{s})=[(1-\eta \kappa) \mathbf{t}+\eta \tau \mathbf{b}](s) .
$$

Differentiating Equation (11) with respect to $s$, we obtain

$$
\begin{gathered}
\ddot{\mathbf{c}}(\bar{s})=\left\{-\eta \dot{\kappa} \mathbf{t}+\left[\kappa-\eta\left(\kappa^{2}-\tau^{2}\right)\right] \mathbf{n}+\eta \dot{\tau} \mathbf{b}\right\}(s) . \\
\dot{\overline{\mathbf{c}}}(\bar{s}) \wedge \ddot{\overline{\mathbf{c}}}(\bar{s})=\left\{-(1-\eta \kappa)\left[\kappa-\eta\left(\kappa^{2}-\tau^{2}\right)\right] \mathbf{b}-\left(\eta \dot{\tau}-\eta^{2} \kappa \dot{\tau}+\eta^{2} \dot{\kappa} \tau\right) \mathbf{n}-\eta \tau\left[\kappa-\eta\left(\kappa^{2}-\tau^{2}\right)\right] \mathbf{t}\right\}(s) . \\
|\dot{\overline{\mathbf{c}}}(\bar{s})|=\left[\eta^{2} \tau^{2}-(1-\eta \kappa)^{2}\right]^{\frac{1}{2}}(s), \\
|\dot{\overline{\mathbf{c}}} \wedge \ddot{\overline{\mathbf{c}}}|(\bar{s})=\left\{\left[\kappa-\eta\left(\kappa^{2}-\tau^{2}\right)\right]^{2}\left[\eta^{2} \tau^{2}-(1-\eta \kappa)^{2}\right]+\left[\eta \dot{\tau}-\eta^{2}(\kappa \dot{\tau}-\dot{\kappa} \tau)\right]^{2}\right\}^{\frac{1}{2}}(s),
\end{gathered}
$$

then put Equations (12) and (13) in Equation (10), we get

$$
\left[\kappa-\eta\left(\kappa^{2}-\tau^{2}\right)\right](s)=\cos \theta \frac{\left\{\left[\kappa-\eta\left(\kappa^{2}-\tau^{2}\right)\right]^{2}\left[\eta^{2} \tau^{2}-(1-\eta \kappa)^{2}\right]+\left[\eta \dot{\tau}-\eta^{2}(\kappa \dot{\tau}-\dot{\kappa} \tau)\right]^{2}\right\}^{\frac{1}{2}}}{\left[\eta^{2} \tau^{2}-(1-\eta \kappa)^{2}\right]^{\frac{1}{2}}}(s) .
$$

Conversely, we will prove that if $\kappa(s)$ and $\tau(s)$ satisfy Equation (14), the principal normal and binormal of $\overline{\mathbf{c}}(\bar{s})$ generated by the equation

$$
\overline{\mathbf{c}}(\bar{s})=\mathbf{c}(s)+\eta(s) \mathbf{n}(s)
$$

are coplanar with the principal normal of $\mathbf{c}(s)$, where $\bar{s}=\bar{s}(s)$. And the angle between $\mathbf{n}(s)$ and $\overline{\mathbf{n}}(\bar{s})$ is $\theta$ in Equation (14), we have known $\eta$ is a constant, then

$$
\overline{\mathbf{t}} \frac{d \bar{s}}{d s}(\bar{s})=[(1-\eta \kappa) \mathbf{t}+\eta \tau \mathbf{b}](s) .
$$

Then we get

$$
\langle\mathbf{n}(s), \overline{\mathbf{t}}(\bar{s})\rangle \frac{d \bar{s}}{d s}=0 .
$$

Since

$$
\frac{d \bar{s}}{d s} \neq 0,
$$

so

$$
\langle\mathbf{n}(s), \overline{\mathbf{t}}(\bar{s})\rangle=0,
$$

so $\mathbf{n}(s)$ is coplanar with $\overline{\mathbf{n}}(\bar{s})$ and $\overline{\mathbf{b}}(\bar{s})$.

Then we prove

$$
\langle\mathbf{n}(s), \overline{\mathbf{n}}(\bar{s})\rangle=\cos \theta .
$$

We suppose

$$
\mathbf{n}(s)=\mu_{1} \overline{\mathbf{n}}(\bar{s})+\mu_{2} \overline{\mathbf{b}}(\bar{s}),
$$


so

$$
\dot{\mathbf{n}}(s)=\left[\mu_{1} \dot{\overline{\mathbf{n}}} \frac{d \bar{s}}{d s}+\mu_{2} \dot{\overline{\mathbf{b}}} \frac{d \bar{s}}{d s}\right](\bar{s}),
$$

and by using the Frenet equations, we have

$$
(-\kappa \mathbf{t}+\tau \mathbf{b})(s)=\left[\mu_{1} \bar{\kappa} \frac{d \bar{s}}{d s} \overline{\mathbf{t}}+\mu_{2} \bar{\tau} \frac{d \bar{s}}{d s} \overline{\mathbf{n}}-\mu_{1} \bar{\tau} \frac{d \bar{s}}{d s} \overline{\mathbf{b}}\right](\bar{s}) .
$$

From Equation (16), we have

$$
\begin{gathered}
{[(1-\eta \kappa) \mathbf{t}+\eta \tau \mathbf{b}](s)=\overline{\mathbf{t}} \frac{d \bar{s}}{d s}(\bar{s}),} \\
{\left[\kappa-\eta\left(\kappa^{2}-\tau^{2}\right)\right](s)=\mu_{1} \bar{\kappa}\left(\frac{d \bar{s}}{d s}\right)^{2}(\bar{s}) .}
\end{gathered}
$$

By the proof of necessity, we can easily obtain Equation (6), then subtract Equations (6) and (19), so we get,

$$
\left(\mu_{1}-\cos \theta\right) \bar{\kappa}\left(\frac{d \bar{s}}{d s}\right)^{2}(\bar{s})=0 .
$$

Since

$$
\left(\frac{d \bar{s}}{d s}\right)^{2} \neq 0, \bar{\kappa}(\bar{s}) \neq 0
$$

so

$$
\mu_{1}=\cos \theta
$$

Then from Equation (17), we can get

$$
\langle\mathbf{n}(s), \overline{\mathbf{n}}(\bar{s})\rangle=\mu_{1}=\cos \theta .
$$

This completes the proof.

Remark 1. In the above Theorem 2 , when $\theta=0$, we have $\cos \theta=1$, then

$$
\left[\kappa-\eta\left(\kappa^{2}-\tau^{2}\right)\right](s)=\frac{\left\{\left[\kappa-\eta\left(\kappa^{2}-\tau^{2}\right)\right]^{2}\left[\eta^{2} \tau^{2}-(1-\eta \kappa)^{2}\right]+\left[\eta \dot{\tau}-\eta^{2}(\kappa \dot{\tau}-\dot{\kappa} \tau)\right]^{2}\right\}^{\frac{1}{2}}}{\left[\eta^{2} \tau^{2}-(1-\eta \kappa)^{2}\right]^{\frac{1}{2}}}(s) .
$$

Squaring both sides of this equation, we obtain

$$
\left[\eta \dot{\tau}-\eta^{2}(\kappa \dot{\tau}-\dot{\kappa} \tau)\right](s)=0,
$$

therefore

$$
\begin{gathered}
{[(\eta \kappa-1) \dot{\tau}](s)=(\eta \dot{\kappa} \tau)(s),} \\
\frac{d \tau}{\tau}=\frac{d \eta \kappa-1}{\eta \kappa-1} .
\end{gathered}
$$

That is, there is a constant $\mu$, such that $\eta \kappa(s)+\mu \tau(s)=1$, this is the necessary and sufficient condition for a curve to be a spacelike Bertrand curve in [4].

Theorem 3. Let $\boldsymbol{c}(s), \overline{\boldsymbol{c}}(\bar{s})$ be two timelike curves with $\boldsymbol{n}(s), \overline{\boldsymbol{n}}(\overline{\mathrm{s}}), \boldsymbol{b}(s)$ and $\overline{\boldsymbol{b}}(\overline{\mathrm{s}})$ being spacelike, then $(c(s), \bar{c}(\bar{s}))$ is a pair of generalized Bertrand curves if and only if the curvature $\kappa(s)$ and torsion $\tau(s)$ of $c(s)$ satisfy

$$
\left[\kappa+\eta\left(\kappa^{2}-\tau^{2}\right)\right](s)=\cos \theta \frac{\left\{\left[\kappa+\eta\left(\kappa^{2}-\tau^{2}\right)\right]^{2}\left[(1+\eta \kappa)^{2}-\eta^{2} \tau^{2}\right]+\left[\eta \dot{\tau}+\eta^{2}(\kappa \dot{\tau}-\dot{\kappa} \tau)\right]^{2}\right\}^{\frac{1}{2}}}{\left[(1+\eta \kappa)^{2}-\eta^{2} \tau^{2}\right]^{\frac{1}{2}}}(s),
$$


where $\theta$ is the angle between $\boldsymbol{n}(s)$ and $\overline{\boldsymbol{n}}(\bar{s}), \eta$ is a non-zero constant, $\dot{\kappa}=\frac{d \kappa}{d s}$ and $\dot{\tau}=\frac{d \tau}{d s}$.

For the other three generalized non-lightlike Bertrand curves in Minkowski 3-space cases, the results are similar, we will not list them here.

\subsection{Generalized Lightlike Bertrand Curves in Minkowski 3-Space}

For lightlike Bertrand curves in $\mathbb{R}_{1}^{3}$, we consider the lightlike Cartan curves here, then the corresponding Frenet frame of its mate curve is divided into the following three conditions: (1) $\mathbf{\mathfrak { t }}(\bar{s})$ and $\overline{\mathbf{b}}(\bar{s})$ are lightlike, $\overline{\mathbf{n}}(\bar{s})$ is spacelike, $(2) \overline{\mathbf{t}}(\bar{s})$ and $\overline{\mathbf{n}}(\bar{s})$ are spacelike, $\overline{\mathbf{b}}(\bar{s})$ is timelike, $(3) \overline{\mathbf{n}}(\bar{s})$ and $\overline{\mathbf{b}}(\bar{s})$ are spacelike, $\overline{\mathbf{n}}(\bar{s})$ is timelike,

Firstly, we consider $\overline{\mathbf{c}}(\bar{s})$ to be a non-lightlike curve, the result is also similar to the generalized non-lightlike Bertrand curve, so we will not list it here. Next we consider $\overline{\mathbf{c}}(\bar{s})$ as a lightlike Cartan curve. Then the normal plane of $\overline{\mathbf{c}}(\bar{s})$ is a lightlike plane, hence when we consider the generalized lightlike Bertrand curves, the angle can not be considered directly by the above method, hence we define it in another way.

Definition 2. Let $\boldsymbol{c}(s)$ and $\overline{\boldsymbol{c}}(\overline{\mathrm{s}})$ be two lightlike Cartan curves in $R_{1}^{3}$, when $\boldsymbol{n}(s)$ and $\overline{\boldsymbol{n}}(\overline{\mathrm{s}})$ span a timelike plane, and satisfy $\langle\boldsymbol{n}(s), \overline{\boldsymbol{n}}(\overline{\mathrm{s}})\rangle=-\cosh \theta$, then we call $\boldsymbol{c}(s)$ a generalized lightlike Bertrand curve, $\overline{\boldsymbol{c}}(\overline{\mathrm{s}})$ a generalized lightlike Bertrand mate and $(\boldsymbol{c}(s), \overline{\boldsymbol{c}}(\overline{\mathrm{s}})$ a pair of generalized lightlike Bertrand curves.

Theorem 4. Let $(\boldsymbol{c}(s), \overline{\boldsymbol{c}}(\bar{s})$ be a pair of generalized lightlike Bertrand curves, then the angle between $\boldsymbol{n}(s)$ and $\bar{n}(\bar{s})$ is $\theta$ if and only if

$$
-\cosh \theta|\ddot{\bar{c}}(s)|=1-2 \eta \tau+\ddot{\eta}-\dot{\eta} \frac{\langle\ddot{\bar{c}}, \dddot{\vec{c}}\rangle}{2|\ddot{\bar{c}}|^{2}}(s),
$$

where $\tau(s)$ is the lightlike curvature of $c(s), \dot{\eta}=\frac{d \eta}{d s}, \ddot{\eta}=\frac{d^{2} \eta}{d s^{2}}, \ddot{\ddot{c}}=\frac{d^{2} \bar{c}}{d s^{2}}$ and $\dddot{\bar{c}}=\frac{d^{3} \bar{c}}{d s^{3}}$.

Proof. As $(\mathbf{c}(s), \overline{\mathbf{c}}(\bar{s}))$ is a pair of generalized lightlike Bertrand curves, then

$$
\overline{\mathbf{c}}(\bar{s})=\mathbf{c}(s)+\eta(s) \mathbf{n}(s),
$$

where $\bar{s}=\bar{s}(s)$, differentiating Equation (20) with respect to $s$, then

$$
\begin{gathered}
\overline{\mathbf{t}} \frac{d \bar{s}}{d s}(\bar{s})=[(1-\eta \tau) \mathbf{t}+\dot{\eta} \mathbf{n}-\eta \mathbf{b}](s), \\
{\left[\overline{\mathbf{n}}\left(\frac{d \bar{s}}{d s}\right)^{2}+\overline{\mathbf{t}} \frac{d^{2} \bar{s}}{d s^{2}}\right](\bar{s})=[(-2 \dot{\eta} \tau-\eta \dot{\tau}) \mathbf{t}+(1-2 \eta \tau+\ddot{\eta}) \mathbf{n}-2 \dot{\eta} \mathbf{b}](s) .}
\end{gathered}
$$

Putting Equation (21) in Equation (22), we can get

$\overline{\mathbf{n}}\left(\frac{d \bar{s}}{d s}\right)^{2}(\bar{s})+[(1-\eta \tau) \mathbf{t}+\dot{\eta} \mathbf{n}-\eta \mathbf{b}] \frac{\frac{d^{2} \bar{s}}{d s^{2}}}{\frac{d \bar{s}}{d s}}(s)=[(-2 \dot{\eta} \tau-\eta \dot{\tau}) \mathbf{t}+(1-2 \eta \tau+\ddot{\eta}) \mathbf{n}-2 \dot{\eta} \dot{b}](s)$.

Taking the pseudo inner product with $\mathbf{n}(s)$ on both sides of Equation (23), we have

$$
-\cosh \theta\left(\frac{d \bar{s}}{d s}\right)^{2}+\dot{\eta} \frac{\frac{d^{2} \bar{s}}{d d^{2}}}{\frac{d \bar{s}}{d s}}(s)=(1-2 \eta \tau+\ddot{\eta})(s) .
$$

Let $\dot{\overline{\mathbf{c}}}=\frac{d \overline{\mathbf{c}}}{d s}=\overline{\mathbf{t}} \frac{d \bar{s}}{d s}$, so

$$
\ddot{\mathbf{c}}(\bar{s})=\left[\overline{\mathbf{n}}\left(\frac{d \bar{s}}{d s}\right)^{2}+\overline{\mathbf{t}} \frac{d^{2} \bar{s}}{d s^{2}}\right](\bar{s}),
$$


then

$$
\langle\ddot{\overline{\mathbf{c}}}(s), \ddot{\overline{\mathbf{c}}}(s)\rangle=\left(\frac{d \bar{s}}{d s}\right)^{4},
$$

hence

$$
\left(\frac{d \bar{s}}{d s}\right)^{2}=|\ddot{\overrightarrow{\mathbf{c}}}(s)| .
$$

Differentiating Equation (25) with respect to $s$, we get

$$
2\langle\ddot{\ddot{\mathbf{c}}}(\bar{s}), \dddot{\mathbf{c}}(\bar{s})\rangle=4\left(\frac{d \bar{s}}{d s}\right)^{3} \cdot \frac{d^{2} \bar{s}}{d s^{2}},
$$

therefore

$$
\frac{d^{2} \bar{s}}{d s^{2}}=\frac{\langle\ddot{\overline{\mathbf{c}}}(\bar{s}), \dddot{\ddot{\mathbf{c}}}(\bar{s})\rangle}{2|\ddot{\overrightarrow{\mathbf{c}}}(\bar{s})|^{\frac{3}{2}}},
$$

putting Equations (26) and (27) in Equation (24), we can obtain

$$
-\cosh \theta|\ddot{\mathbf{c}}(\bar{s})|=1-2 \eta \tau+\ddot{\eta}-\dot{\eta} \frac{\langle\ddot{\ddot{\mathbf{c}}}, \dddot{\overrightarrow{\mathbf{c}}}\rangle}{2|\ddot{\overrightarrow{\mathbf{c}}}|^{2}}(s) .
$$

Conversely, we will prove that if the lightlike curvature $\tau(s)$ of the curve $\mathbf{c}(s)$ satisfies Equation (28), then the curve generated by the equation

$$
\overline{\mathbf{c}}(\bar{s})=\mathbf{c}(s)+\eta(s) \mathbf{n}(s)
$$

is a generalized lightlike Bertrand mate, where $\bar{s}=\bar{s}(s)$. And the angle between $\mathbf{n}(s)$ and $\overline{\mathbf{n}}(\bar{s})$ is $\theta$ in Equation (28). Differentiating Equation (29) with respect to $s$, we obtain

$$
\begin{gathered}
\overline{\mathbf{t}} \frac{d \bar{s}}{d s}(\bar{s})=[(1-\eta \tau) \mathbf{t}+\dot{\eta} \mathbf{n}-\eta \mathbf{b}](s), \\
{\left[\overline{\mathbf{n}}\left(\frac{d \bar{s}}{d s}\right)^{2}+\overline{\mathbf{t}} \frac{d^{2} \bar{s}}{d s^{2}}\right](\bar{s})=[(-2 \dot{\eta} \tau-\eta \dot{\tau}) \mathbf{t}+(1-2 \eta \tau+\ddot{\eta}) \mathbf{n}-2 \dot{\eta} \mathbf{b}](s),}
\end{gathered}
$$

putting Equation (30) in Equation (31), we have

$$
\overline{\mathbf{n}}\left(\frac{d \bar{s}}{d s}\right)^{2}(\bar{s})+[(1-\eta \tau) \mathbf{t}+\dot{\eta} \mathbf{n}-\eta \mathbf{b}] \frac{\frac{d^{2} \bar{s}}{d s^{2}}}{\frac{d \bar{s}}{d s}}(s)=[(-2 \dot{\eta} \tau-\eta \dot{\tau}) \mathbf{t}+(1-2 \eta \tau+\ddot{\eta}) \mathbf{n}-2 \dot{\eta} \mathbf{b}](s) .
$$

so

$$
\langle\overline{\mathbf{n}}(\bar{s}), \mathbf{n}(s)\rangle\left(\frac{d \bar{s}}{d s}\right)^{2}+\dot{\eta}(s) \frac{\frac{d^{2} \bar{s}}{d s^{2}}}{\frac{d \bar{s}}{d s}}=(1-2 \eta \tau+\ddot{\eta})(s) .
$$

By the proof of necessity, we can easily obtain Equation (24), then subtract Equations (24) and (33), and we have

$$
(\cosh \theta+\langle\overline{\mathbf{n}}(\bar{s}), \mathbf{n}(s)\rangle)\left(\frac{d \bar{s}}{d s}\right)^{2}=0 .
$$

Since

$$
\left(\frac{d \bar{s}}{d s}\right)^{2} \neq 0
$$

so

$$
\langle\mathbf{n}(s), \overline{\mathbf{n}}(\overline{\mathbf{s}})\rangle=-\cosh \theta .
$$

This completes the proof.

In [6], Honda and Inoguchi gave the necessary and sufficient condition for a curve to be a lightlike Bertrand curve. 
Theorem 5. Let $c(s)$ be a lightlike Cartan curve, then $\boldsymbol{c}(s)$ is a lightlike Bertrand curve if and only if its lightlike curvature $\tau=\frac{1}{\eta}$, where $\eta$ is a non-zero constant (see [6]).

Remark 2. In the Theorem 4, when $\theta=0$, we have $\cosh \theta=1$. In this case, $(\boldsymbol{c}(s), \overline{\boldsymbol{c}}(\overline{\mathrm{s}}))$ is a pair of lightlike Bertrand curves, so $\tau=\frac{1}{\eta}$ is a constant, then the left side of Equation (28) is -1 . When $(\boldsymbol{c}(s), \overline{\boldsymbol{c}}(\bar{s}))$ is a pair of lightlike Bertrand curves, we have

$$
\left(\frac{d \bar{s}}{d s}\right)^{2}=1
$$

so

$$
|\ddot{\overrightarrow{\boldsymbol{c}}}(\bar{s})|=1,
$$

then the right side of Equation (28) is -1. Hence the left is equal to the right of Equation (28), that is when $(c(s), \bar{c}(\bar{s}))$ is a pair of lightlike Bertrand curves, the Theorem 4 still holds.

\section{The Relationship between a Slant Helix and a Generalized Bertrand Curve in Minkowski 3-Space}

Proposition 1. Let $c=c(s): I \rightarrow \mathbb{R}_{1}^{3}$ be a non-lightlike curve, then it is a non-lightlike slant helix if and only if

$$
\varphi(s)=\frac{-\delta(\mathbf{t}) \delta(\mathbf{d}) \kappa^{2}\left(\delta(\mathbf{n})\left(\frac{\tau}{\kappa}\right)\right)}{\left[\delta(\mathbf{t}) \delta(\mathbf{d})\left(\tau^{2}-\delta(\mathbf{n}) \kappa^{2}\right)\right]^{\frac{3}{2}}}(s)
$$

is a constant function, where $\tau^{2}-\delta(\mathbf{n}) \kappa^{2} \neq 0$.

Proposition 2. Let $c=c(s): I \rightarrow \mathbb{R}_{1}^{3}$ be a lightlike Cartan curve, then it is a Cartan slant helix if and only if

$$
\psi(s)=\frac{-\dot{\tau}}{2 \sqrt{2}|\tau|^{\frac{3}{2}}}(s)
$$

is a constant function (see [21]).

Theorem 6. Let $\boldsymbol{c}(s)$ be a spacelike slant helix with $\boldsymbol{n}(s)$ being spacelike and $\boldsymbol{b}(s)$ being timelike in $\mathbb{R}_{1}^{3}$, then $\boldsymbol{c}(s)$ is a generalized Bertrand curve if and only if there exist constants $\eta, \mu$ such that its curvature $\kappa(s)$ and torsion $\tau(s)$ satisfy

$$
\delta(\mathbf{d}) \eta \dot{\tau}(s)+\eta^{2} \mu M^{3}(s)=\left[\delta(\mathbf{d}) \kappa(s)+\eta M^{2}(s)\right]\left[\eta^{2} \tau^{2}(s)-(1-\eta \kappa(s))^{2}\right]^{\frac{1}{2}} \tan \theta,
$$

where $M^{2}(s)=\delta(\mathbf{d})\left[\tau^{2}(s)-\kappa^{2}(s)\right], \theta$ is the angle between the prinpical normal lines of the generalized Bertrand curve and its generalized timelike Bertrand mate.

Proof. As $\mathbf{c}(s)$ is a spacelike slant helix in $\mathbb{R}_{1}^{3}$, by Proposition 1 , we obtain $\mathbf{c}(s)$ to be a spacelike slant helix if and only if

$$
\varphi(s)=\frac{-\delta(\mathbf{d}) \kappa^{2}}{\left[\delta(\mathbf{d})\left(\tau^{2}-\kappa^{2}\right)\right]^{\frac{3}{2}}}\left(\frac{\tau}{\kappa}\right) \cdot(s)
$$

is a constant function.

Let $\varphi(s)=\mu, \mu$ is a constant, $M^{2}(s)=\delta(\mathbf{d})\left[\tau^{2}(s)-\kappa^{2}(s)\right]$, then

$$
\dot{\kappa}(s) \tau(s)-\kappa(s) \dot{\tau}(s)=\frac{\mu M^{3}(s)}{\delta(\mathbf{d})},
$$

by Theorem 2, c(s) is a generalized Bertrand curve if and only if

$$
\left[\kappa-\eta\left(\kappa^{2}-\tau^{2}\right)\right](s)=\cos \theta \frac{\left\{\left[\kappa-\eta\left(\kappa^{2}-\tau^{2}\right)\right]^{2}\left[\eta^{2} \tau^{2}-(1-\eta \kappa)^{2}\right]+\left[\eta \dot{\tau}-\eta^{2}(\kappa \dot{\tau}-\dot{\kappa} \tau)\right]^{2}\right\}^{\frac{1}{2}}}{\left[\eta^{2} \tau^{2}-(1-\eta \kappa)^{2}\right]^{\frac{1}{2}}}(s),
$$


Then we can easily obtain that

$$
1+\frac{\left[\eta \dot{\tau}-\eta^{2}(\kappa \dot{\tau}-\dot{\kappa} \tau)\right]^{2}}{\left[\kappa-\eta\left(\kappa^{2}-\tau^{2}\right)\right]^{2}\left[\eta^{2} \tau^{2}-(1-\eta \kappa)^{2}\right]}(s)=1+\tan ^{2} \theta .
$$

Since the slant helix is a generalized Bertrand curve, we put Equation (35) in Equation (36), and we get

$$
\delta(\mathbf{d}) \eta \dot{\tau}(s)+\eta^{2} \mu M^{3}(s)=\left[\delta(\mathbf{d}) \kappa(s)+\eta M^{2}(s)\right]\left[\eta^{2} \tau^{2}(s)-(1-\eta \kappa(s))^{2}\right]^{\frac{1}{2}} \tan \theta .
$$

Conversely, let $\mathbf{c}(s)$ be a spacelike slant helix, its $\kappa(s)$ and $\tau(s)$ satisfy Equation (34), where $M^{2}(s)=\delta(\mathbf{d})\left[\tau^{2}(s)-\kappa^{2}(s)\right]$. Let $\mu=\varphi(s)$, then Equation (34) can be write

$$
\left[\kappa-\eta\left(\kappa^{2}-\tau^{2}\right)\right](s)=\cos \theta \frac{\left\{\left[\kappa-\eta\left(\kappa^{2}-\tau^{2}\right)\right]^{2}\left[\eta^{2} \tau^{2}-(1-\eta \kappa)^{2}\right]+\left[\eta \dot{\tau}-\eta^{2}(\kappa \dot{\tau}-\dot{\kappa} \tau)\right]^{2}\right\}^{\frac{1}{2}}}{\left[\eta^{2} \tau^{2}-(1-\eta \kappa)^{2}\right]^{\frac{1}{2}}}(s),
$$

hence this slant helix is a generalized Bertrand curve. This completes the proof.

Theorem 7. Let $\boldsymbol{c}(s)$ be a timelike slant helix with $\boldsymbol{n}(s)$ and $\boldsymbol{b}(s)$ being spacelike in $\mathbb{R}_{1}^{3}$, then $\boldsymbol{c}(s)$ is a generalized Bertrand curve if and only if there exist constants $\eta, \mu$ such that its curvature $\kappa(s)$ and torsion $\tau(s)$ satisfy

$$
\delta(\mathbf{d}) \eta \dot{\tau}(s)-\eta^{2} \mu M^{3}(s)=\left[\delta(\mathbf{d}) \kappa(s)-\eta M^{2}(s)\right]\left[(1+\eta \kappa(s))^{2}-\eta^{2} \tau^{2}(s)\right]^{\frac{1}{2}} \tan \theta,
$$

where $M^{2}(s)=\delta(\mathbf{d})\left[\kappa^{2}(s)-\tau^{2}(s)\right], \theta$ is the angle between the principal normal lines of the generalized Bertrand curve and its generalized timelike Bertrand mate.

Theorem 8. Let $\boldsymbol{c}(s)$ be a lightlike slant helix with $\boldsymbol{n}(s)$ being spacelike and $\boldsymbol{b}(s)$ being lightlike, then $\boldsymbol{c}(s)$ is a generalized lightlike Bertrand curve if and only if there exist constants $\eta, \mu$ such that its lightlike curvature $\tau(s)$ satisfies

$$
4 \eta^{3} \mu^{2}|\tau|^{3}(s)=(1-\eta \tau(s))(1-2 \eta \tau(s))^{2} \tan ^{2} \theta,
$$

where $\theta$ is the angle between the principal normal lines of the generalized lightlike Bertrand curve and its generalized timelike Bertrand mate.

\section{Conclusions}

In conclusion, we define the generalized Bertrand curve by considering that the principal normal of one curve falls on the normal plane of another curve, and there is a certain angle between the principal normal lines of two curves. We obtain sufficient and necessary condition for a curve to be a generalized Bertrand curve, and a slant helix can become a generalized Bertrand curve when its curvature and torsion satisfy some conditions. Because of the existence of this angle, our results are more generalized. Hence, the generalized Bertrand curve defined by us will be widely used in mathematics and other fields, such as in the field of biochemistry, road edge information extraction from high-resolution remote sensing images, the method of curve generation in computer aided geometric design (see [12-18]). With the rapid development of computer technology, this has very important theoretical and practical significance.

Author Contributions: Writing-Original Draft Preparation, C.Z.; Writing-Review and Editing, D.P.; Funding Acquisition, D.P. All authors have read and agreed to the published version of the manuscript.

Funding: This research was funded by the National Natural Science Foundation of China grant number 11671070.

Acknowledgments: The authors would like to thank the reviewers for their careful reading and constructive suggestions.

Conflicts of Interest: The authors declare no conflict of interest. 


\section{References}

1. Jie, H.; Pei, D. Singular Special Curves in 3-Space Forms. Mathematics 2020, 8, 846.

2. Izumiya, S.; Takeuchi, N. Generic properties of helices and Bertrand curves. J. Geom. 2002, 74, 97-109. [CrossRef]

3. Ekmekçi, N.; İlarslan, K. On Bertrand Curves and their characterization. Differ. Geom. Dyn. Syst. 2001, 3, 17-24.

4. Balgetir, H.; Bektaşc, M.; Ergüt, M. Bertrand curves for nonnull curves in 3-dimensional Lorentzian space. Hadron. J. 2004, 27, 229-236.

5. Balgetir, H.; Bektaşc, M.; Inoguchi, J. Null Bertrand Curves in Minkowski 3-Space and their characterizations. Note Mat. 2004, 23, 7-13.

6. Honda, K.; Inoguchi, J. Deformations of Cartan framed null curves preserving the torsion. Differ. Geom. Dyn. Syst. 2003, 5, 31-37.

7. Lucas, P.; Ortega-Yagües, J.A. Bertrand curves in the three-dimensional sphere. J. Geom. Phys. 2012, 62, 1903-1914. [CrossRef]

8. Lucas, P.; Ortega-Yagües, J.A. Bertrand Curves In Non-Flat 3-Dimensional (Riemannian and Lorentzian) Space forms. J. Bull. Korean Math. Soc. 2013, 50, 1109-1126. [CrossRef]

9. Yildirim, M.; Bektaş, M. General properties of Bertrand curves in Riemann-Otsuki space. Nonlinear Anal. 2008, 69, 3225-3231. [CrossRef]

10. Choi, J.H.; Kang, T.H.; Kim, Y.H. Bertrand curves in 3-dimensional space forms. Appl. Math. Comput. 2012, 219, 1040-1046. [CrossRef]

11. Şenol, A.; Ziplar, E.; Yayli, Y. General helices and Bertrand curves in Riemannian space form. Math. Aeterna 2012, 2, 155-161.

12. Watson, J.D.; Crick, F.H. Molecular structures of nucleic acids. Nature 1953, 171, 737-738. [CrossRef]

13. Jain, A.; Wang, G.; Vasquez, K.M. DNA triple helices: Biological consequences and the therapeutic potential. Biochemie 2008, 90, 1117-1130. [CrossRef]

14. Jäntschi, L. The Eigenproblem Translated for Alignment of Molecules. Symmetry 2019, 11, 1027. [CrossRef]

15. Jäntschi, L.; Bolboaca, S.D. Study of geometrical shaping of linear chained polymers stabilized as helixes. Stud. Univ.-Babes-Bolyai Chem. 2016, 61, 123-136.

16. Papaioannou, S.G.; Kiritsis, D. An application of Bertrand curves and surface to CAD/CAM. Comput. Aided Des. 1985, 17, 348-352. [CrossRef]

17. Ravani, B.; Ku, T.S. Bertrand offsets of ruled and developable surfaces. Comput. Aided Des. 1991, 23, 145-152. [CrossRef]

18. Amini, J.; Saradjian, M.R.; Blais, J.A.R.; Lucas, C.; Azizi, A. Automatic road-side extraction from large scale imagemaps. Int. J. Appl. Earth. Obs. 2002, 4, 95-107. [CrossRef]

19. Bonnor, W.B. Null curves in Minkowski space-time. Tensor 1969, 20, 229-242.

20. Izumiya, S.; Takiyama, A. A time-like surface in Minkowski 3-space which contains pseudocircles. Proc. Edinb. Math. Soc. 1997, 40, 127-136. [CrossRef]

21. Liu, T.; Pei, D. Null helices and Cartan slant helices in Lorentz-Minkowski 3-space. Int. J. Geom. Methods Mod. Phys. 2019, 16, 1950179. [CrossRef]

Publisher's Note: MDPI stays neutral with regard to jurisdictional claims in published maps and institutional affiliations.

(C) 2020 by the authors. Licensee MDPI, Basel, Switzerland. This article is an open access article distributed under the terms and conditions of the Creative Commons Attribution (CC BY) license (http://creativecommons.org/licenses/by/4.0/). 